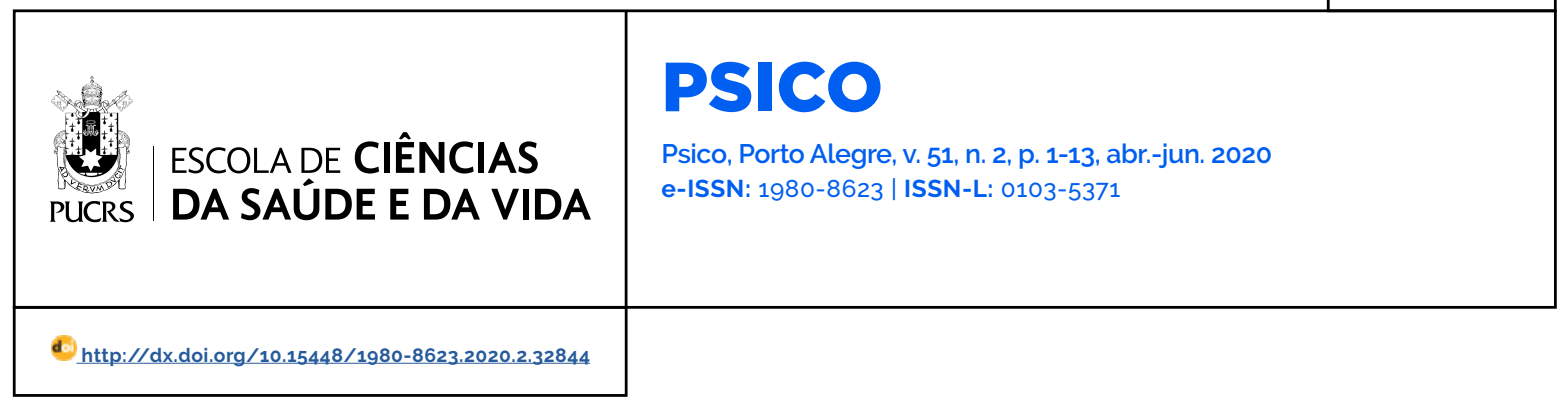

\title{
A brincadeira como promotora da habilidade de comunicação intencional infantil: uma revisão sistemática
}

\author{
The play as a promoter of the ability of intentional child communication: a systematic review
}

La broma como promotora de la habilidad de comunicación intencional infantil: una revisión sistemática

\section{Ana Cristina Ramos \\ Costa ${ }^{1}$}

orcid.org/0000-0002-5194-199X

anacristinaramoscosta@gmail.com

\section{Vanessa Porto \\ Alexandrino ${ }^{1}$ \\ orcid.org/0000-0002-7822-6830 \\ vanessaporto.alexandrino@ \\ gmail.com}

\section{Jéssica Andrade de \\ Albuquerque ${ }^{1}$}

orcid.org/0000-0002-6833-3982

jessica.a.psi@gmail.com

\section{Fabiola de Sousa Braz \\ Aquino $^{1}$}

orcid.org/0000-0002-8854-8577

fabiolabrazaquino@gmail.com

fabiolabrazaquino@cchla.ufpb.br

Recebido em: 21 dez. 2018

Aprovado em: 25 jul. 2019

Publicado em: 4 set. 2020

\section{(c) (1)}

Artigo está licenciado sob forma de uma licenç Creative Commons Atribuição 4.0 Internacional.
Resumo: Esse estudo apresenta uma revisão sistemática de pesquisas que abordam a atividade de brincadeira conjunta em interações mãe-bebê como favorecedora da habilidade de comunicação intencional infantil. Essa revisão seguiu as recomendações metodológicas do Preferred Reporting Items for Systematic reviews and Meta-Analyse (Prisma) e teve as buscas realizadas nas bases de dados Web of Science, Pubmed, Pepsic, Scielo, Indexpsi, PsycInfo e Lilacs e, também, na Biblioteca Digital Brasileira de Teses e Dissertações (BDTD). Os descritores utilizados foram mother, baby, communication, intentionality e play. A análise permitiu incluir 10 artigos e uma tese de doutorado que foram publicados entre 2008 e 2018. A pesquisa permitiu identificar a necessidade de ampliar os estudos sobre o tema com pesquisas que investiguem estilos interativos de bebês com adultos em diferentes grupos sociais, contextos diversos de aprendizado e tipos de brincadeira que podem fomentar o desenvolvimento de habilidades de comunicação intencional. Palavras-chave: brincadeira, comunicação, intencionalidade.

Abstract: This study presents a systematic review of research that addresses the joint play activity in mother-infant interactions as a facilitator of the intentional child communication ability. This review followed the methodological recommendations of the Preferred Reporting Items for Systematic Reviews and Meta-Analyze / PRISMA and has been searched in the Web of Science, Pubmed, Pepsic, Scielo, Indexpsi, Psyclnfo and Lilacs databases and also in the Brazilian Digital Library of Theses and Dissertations (BDTD). The descriptors used were "mother", "baby", "communication", "intentionality" and "play". The analysis allowed to include 10 articles and a doctoral thesis that were published between 2008 and 2018. The research allowed to identify the need to broaden the studies on the subject with research investigating interactive styles of infants with adults in different social groups, contexts other than learning. and types of play that can foster the development of intentional communication skills. Keywords: play, communication, intentionality.

Resumen: Este estudio presenta una revisión sistemática de investigaciones que abordan la actividad de juego conjunta en interacciones madre-bebé como favorecedora de la habilidad de comunicación intencional infantil. Esta revisión siguió las recomendaciones metodológicas del Preferred Reporting Items para las evaluaciones de diagnóstico y Meta-Analyse / PRISMA y tuvo las búsquedas realizadas en las bases de datos Web of Science, Pubmed, Pepsic, Scielo, Indexpsi, Psyclnfo y Lilacs y también en la Biblioteca Digital Brasileña de Tesis y Disertaciones (BDTD). Los descriptores utilizados fueron "mother", "baby", "communication", "intencionality" y "play". El análisis permitió incluir 10 artículos y una tesis de doctorado que se publicaron entre 2008 y 2018. La investigación permitió identificar la necesidad de ampliar los estudios sobre el tema con investigaciones que investiguen estilos interactivos de bebés con adultos en diferentes grupos sociales, contextos diversos aprendizaje, y tipos de juego que pueden fomentar el desarrollo de habilidades de comunicación intencional. Palabras clave: broma, la comunicación, intencionalidad. 
O presente artigo é parte de um estudo mais amplo de dissertação que investigou as configurações da habilidade comunicativa intencional de bebês em três periodos dos dois primeiros anos de vida, em contexto de brincadeira, como foco na identificação de episódios que evidenciassem o uso da comunicação intencional pelos bebês durante a atividade de brincadeira conjunta, aqui entendida como uma atividade que promove o desenvolvimento desse tipo de comunicação. Segundo Tomasello e Carpenter (2007), a relevância do estudo aprofundado dessas habilidades reside no fato de os seres humanos se caracterizarem como os únicos no reino animal que possuem a habilidade de se comunicar com seus coespecificos de forma intencionalmente comunicativa. Estudos no campo da Psicologia do Desenvolvimento (Rivero, 2003; Rochat, 2007; Striano \& Vaish, 2006) têm abordado os processos cognitivos e motivacionais que promovem uma maior compreensão acerca das primeiras manifestações da capacidade intencional dos bebês.

Uma das atividades mais importantes e peculiares dos seres humanos, segundo Hamann, Warneken e Tomasello (2012), refere-se à habilidade de estabelecer ações conjuntas de forma intencional e compartilhada. A habilidade de comunicação intencional é definida pela coordenação, por parte da criança, das ações dirigidas a um objeto externo e a outra pessoa, demandando da criança a comunicação de intenções sobre os objetos para outros indivíduos que podem ajudá-la a satisfazer seus objetivos (Riveiro; 2003; Sarriá, 1991). Esses comportamentos podem ser percebidos em interações face a face mãe-bebê, principalmente, a partir dos seis meses de vida da criança (Aquino \& Salomão, 2011a; Tomasello, 2003) por meio de gestos de pegar objetos oferecidos pela mãe e/ou dar objetos espontaneamente (Papaeliou \& Trevarthen, 2006), apontar para objetos e alternar o olhar entre um objeto e um sujeito da interação (Sarriá, 1991).

A habilidade de comunicação intencional nesse artigo será abordada considerando um contexto específico de ação conjunta estabelecida entre diades mãe-bebê, qual seja, a situação de brincadeira. Vigotsky (2004) defendeu que a brincadeira é uma atividade cultural e o caminho principal do desenvolvimento nos primeiros anos de vida. Para esse autor, a brincadeira contém em si, "como na mágica de uma lente de aumento, todas as tendências do desenvolvimento" ( $p$. 35), o que a coloca em uma situação de análise privilegiada e como elemento fundamental para o desenvolvimento na primeira infância.

Pesquisadores contemporâneos da Psicologia do Desenvolvimento (Mendes \& Seidl de Moura, 2004; Queiroz, Maciel, \& Branco, 2006) ratificam a concepção vygotskyana segundo a qual a atividade de brincar é marcada pela experiência com a cultura, tornando-se o elemento de mediação que integra o sistema de funções psicológicas por meio dos processos de interação, utilizando recursos e instrumentos semióticos coconstruidos de uma geração mais experiente com a qual a criança entra em contato. Assim, tais autores reconhecem a importância da brincadeira para o desenvolvimento infantil por permitir que a criança construa a experiência de se relacionar com o mundo de maneira ativa, podendo transformar e produzir novos significados.

Estudos mais recentes continuam sustentando a ideia de que a brincadeira é um fator vital para o desenvolvimento de habilidades tipicamente humanas, como a cognição, a afetividade, a motricidade e a dimensão sociocomunicativa (Cypel, 2011; Smirnova \& Riabkova, 2016; Villachan-Lyra, Queiroz, Moura, \& Gil, 2018). Por meio da brincadeira, a criança encontra formas para elaborar vivências emocionais significativas, estimular a cognição e, também, se comunicar (Teodoro, 2013). Além disso, a brincadeira cria condições ideais para o desenvolvimento dos diversos aspectos da personalidade das crianças, incluindo as vivências delas em contextos escolares (Smirnova \& Riabkova, 2016).

Considerando os argumentos anteriores, este trabalho enfatiza o papel da situação de brincadeira como um contexto interativo privilegiado para a ocorrência de mediações e de trocas intersubjetivas que favorecem o desenvolvimento de habilidades sociocognitivas e linguísticas infantis, como a habilidade de 
comunicação intencional. Por isso, no intuito de colaborar para discussões referentes ao desenvolvimento de bebês nos primeiros anos de vida, e para ampliar a compreensão acerca das modalidades de comportamento intencional e comunicativo em interações mãe-bebê no contexto de brincadeira, foi realizada uma revisão sistemática que teve como objetivo identificar estudos que abordassem a brincadeira como favorecedora da habilidade de comunicação intencional infantil.

\section{Método}

O presente estudo trata-se de uma revisão sistemática. Para Almeida e Goulart (2017) revisões sistemáticas utilizam como fonte de dados a literatura sobre um tema, através de uma síntese das evidências a partir de estratégias especificas de métodos sistematizados de busca, análise crítica e resumo dos estudos localizados. A presente revisão seguiu as recomendações do Preferred Reporting Items for Systematic reviews and MetaAnalyse (Prisma) (Moher, Liberati, Tetzlaff, Altman, \& The Prisma group, 2015) e está registrada no banco de dados internacional de protocolos de revisão sistemática International Prospective Register of Ongoing Systematic Reviews (Prospero) sob o número CRD42018110629.

Para selecionar as produções que abordavam a temática da brincadeira como promotora da comunicação intencional foi realizada uma busca eletrônica abrangente nas bases de dados Web of Science, Pubmed, Pepsic, Scielo, Indexpsi, Psyclnfo e Lilacs. Também foi realizada uma busca na Biblioteca Digital Brasileira de Teses e Dissertações (BDTD) como forma de ampliar as buscas por produções sobre a temática. A data da última busca foi em 24 de setembro de 2018. Os descritores utilizados (com base em uma pesquisa junto aos Descritores em Ciências da Saúde- DeCs) foram mother, baby, communication, intentionality e play.

A estratégia de busca eletrônica nas bases de dados foi realizada a partir da construção do PICO para organizar os termos e cruzamento dos descritores, sendo $\mathrm{P}=$ (population) mother or baby, I= (intervention) play, $\mathrm{C}=$ (comparation) não foi incluido termo, por não englobar comparações nos estudos que se propunham localizar, e $\mathrm{O}=$ (outcome) communication or intentionality. Os referidos descritores foram cruzados em todas as bases de dados da mesma maneira conforme a organização do PICO.

O PICO auxilia na definição da pergunta de pesquisa e na organização da busca por produções na literatura, a partir do arranjo dos descritores. Tal estratégia possibilita maximizar o alcance da busca por publicações acadêmicas, evita buscas dispensáveis e permite ao pesquisador o rápido acesso às informações científicas disponiveis sobre o tema pesquisado (Santos, Pimenta, \& Nobre, 2007).

\section{Critérios de elegibilidade}

Os critérios elegiveis para identificar as produções disponiveis na literatura que abordam a brincadeira como promotora de comunicação intencional foram: (1) artigos com qualquer tipo de delineamento de pesquisa (exceto estudos de revisão); (2) artigos redigidos em qualquer idioma; (3) artigos cujos participantes fossem mães e bebês com desenvolvimento típico; e (4) ser artigo original de pesquisa desenvolvida com seres humanos, publicado em periódico indexado nas bases utilizadas, entre janeiro de 2008 a setembro de 2018.

\section{Seleção dos estudos}

Segundo Almeida e Goulart (2017, p. 554) "é comum que no processo de extração de dados em revisões sistemáticas tenham dois revisores coletando as informações dos estudos primários de forma independente, (...) resolvendo as discordâncias com um terceiro revisor ou por meio de um consenso". Nesse sentido, primeiramente, duas pesquisadoras avaliaram de forma independente os titulos e resumos das produções das bases de dados para a seleção de estudos potencialmente elegiveis. Desses, foram buscados os textos completos para a avaliação minuciosa, dos quais foram incluídos os estudos que atenderam aos critérios de elegibilidade previamente estabelecidos. Nos casos de discordância, foi contatado um terceiro avaliador. 


\section{Processo de coleta de dados}

Foi criado um formulário para a coleta dos dados dos artigos incluidos. O referido formulário englobou os seguintes itens: título, desenho e ano do estudo, participantes, objetivos e principais resultados. Esse processo foi realizado por duas pesquisadoras de forma independente. Para analisar os resultados obtidos nesta revisão, optou-se por organizar os estudos selecionados nos seguintes eixos de análise: data e periódicos; autoria e vinculo institucional; objetivo dos estudos; delineamento das pesquisas, amostras e instrumentos; e principais resultados.

\section{Risco de viés dos estudos}

A análise do risco de viés dos estudos foi realizada através do instrumento Risk Of Bias In Non-randomized Studies-of Interventions (ROBINS-I). Essa é uma ferramenta Cochrane para avaliação da qualidade metodológica dos estudos observacionais, a qual contém sete dominios que são classificados em domínios pré-intervenção e pós-intervenção para estudos observacionais, quais sejam: confundimento, seleção dos participantes, aferição da intervenção (exposição), recebimento da intervenção atribuida, perdas, aferição nos desfechos e relato seletivo dos desfechos. Os referidos domínios foram avaliados por duas pesquisadoras de forma independente.

\section{Resultados e discussão}

As primeiras buscas levaram aos seguintes resultados no que se refere à quantidade de publicações: Web of Science, 23 artigos; Psycinfo, 340 artigos; Pubmed, 295 artigos; Lilacs,15 artigos; SciELO, 7 artigos; IndexPsi, 4 artigos; Pepsic, 3 artigos; e Scopus: 677 artigos. Na Biblioteca Digital Brasileira de Teses e Dissertações foram localizadas 20 produções. Após a leitura dos títulos e resumos, restaram as seguintes produções: Web of Science, 4 artigos; Psyclnfo, 4 artigos; Pubmed, 3 artigos; Lilacs, 4 artigos; SciELO, 3 artigos; IndexPsi, 2 artigos; Pepsic, 1 artigo; Scopus, 2 artigos; e BDTD, 2 produções. Por fim, após a leitura das produções na integra, a seleção das produções se restringiu aos seguintes números: Psyclnfo, 1; Pubmed, 3; Lilacs, 4; Pepsic, 1; Scopus, 1; e BDTD, 1, totalizando 10 artigos e uma tese de doutorado, ou seja, 11 produções que foram analisadas neste estudo de revisão sistemática. O fluxograma da seleção das publicações pode ser visualizado na Figura 1.

Figura 1 - Fluxograma de seleção das publicações

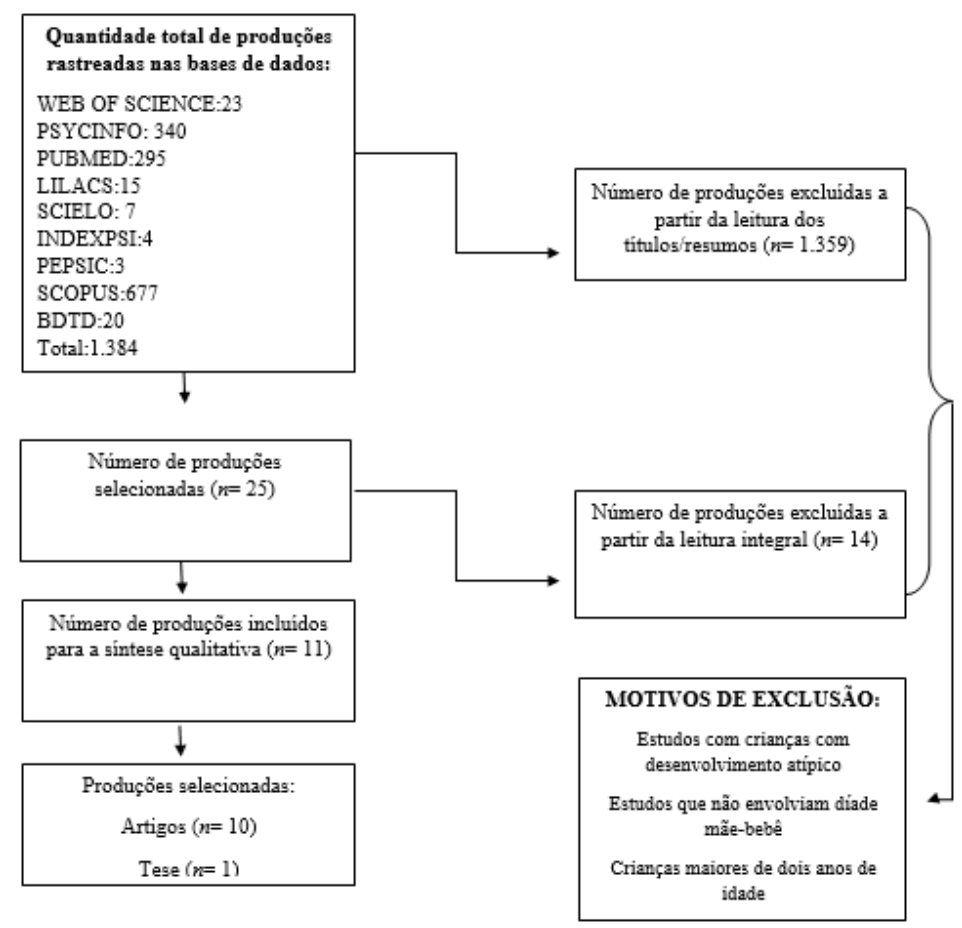




\section{Data das publicações e periódicos}

A análise das publicações indicou que dentro do recorte de 10 anos (2008-2018), escolhido para essa revisão, por se buscar verificar o panorama mais atual de estudos sobre o tema, as publicações sobre a brincadeira como favorecedora do desenvolvimento da habilidade de comunicação intencional de bebês teve maior número de publicações nos anos de 2011, 2012 e 2017, revelando um crescimento nos últimos anos.

No que se refere aos periódicos, foi possivel perceber que seis dos estudos localizados foram publicados em periódicos de âmbito nacional, enquanto cinco foram publicados em âmbito internacional. Os periódicos internacionais variaram entre aqueles do campo da Psicologia (Developmental Neuropsychology), dos estudos sobre a infância (Infant) e Infant Behavior and Development, e dos estudos sobre a linguagem: (First Language). Dos estudos selecionados em periódicos de âmbito nacional $(n=6)$, quatro eram periódicos da área da Psicologia: Psicologia Usp; Estudos e Pesquisas em Psicologia; e Psicologia: Reflexão e Crítica. Um dos artigos localizados foi publicado em periódico da área da Filosofia, Ciências e Letras: Paidéia. A tese de doutorado foi localizada pela Biblioteca Brasileira de Teses e Dissertações (BDTD) e foi desenvolvida na área da Psicologia Social.

Essas informações nos permitem o entendimento de que a temática da brincadeira como favorecedora da habilidade da comunicação intencional dos bebês tem recebido destaque e crescimento nos últimos anos, o que pode indicar, além de um maior interesse dos pesquisadores nesse âmbito, a possibilidade de um aprofundamento teórico sobre o tema, que pode gerar uma reconfiguração nas interações com os bebês tanto em ambientes familiares como em espaços de educação formal. Sobre o número de produções nos âmbitos nacional e internacional, considera-se escasso de maneira geral. No entanto, ressalta-se que o número de estudos desenvolvidos no Brasil foi significativo e nos revela que a temática tem sido abordada com vistas a produzir resultados que possam ser reproduzidos pela comunidade em geral, informando a importância da brincadeira para o desenvolvimento.
No que se refere ao maior número de publicações em periódicos da área da Psicologia, ressaltamos a relevância dessa ciência estar atenta às questões do desenvolvimento infantil nos períodos iniciais, haja vista que é parte de sua área de conhecimento o estudo e formulação de recomendações para suas contribuições no desenvolvimento humano e em intervenções nesse campo. Segundo Aquino e Salomão (2011a), os estudos sobre o desenvolvimento humano inicial são relevantes, pois dão importantes indicadores sobre a gênese do desenvolvimento humano e favorecem o entendimento sobre as relações entre a comunicação não verbal, a intencionalidade sociocomunicativa e a aquisição da linguagem. Para Tomasello (2003), as interações sociais entre adulto e criança, desde os primeiros meses de vida, dão suporte para a construção de relações entre o bebê e a cultura na qual está inserido, através de trocas intersubjetivas adulto-bebê que permitem novas formas de representação cognitiva sobre o meio social.

\section{Autoria e vínculo institucional}

No que se refere à autoria, todos os artigos localizados $(n=10)$ foram de autoria coletiva, sendo apenas a tese de doutorado o estudo de autoria única (Nogueira, 2009). Os artigos publicados em âmbito internacional exploravam autores de paises como Estados Unidos, França, Holanda e Austrália. Dois artigos, embora publicados em periódicos brasileiros, apresentavam autores de Portugal. Das produções localizadas em âmbito nacional, dois artigos tinham apenas autoras do nordeste brasileiro, enquanto a tese de doutorado foi desenvolvida por uma pesquisadora do Estado do Rio de Janeiro.

No tocante às informações acerca da autoria coletiva e local das publicações, essas nos permitem observar dois pontos importantes: o primeiro que em estudos observacionais a presença de mais de um observador/ pesquisador é relevante para a minimização de vieses. As pesquisas localizadas demonstram a preocupação em estabelecer corpo de juizes e avaliação dos resultados por, no mínimo, dois pesquisadores. Essa tendência parece constituir 
um padrão nesse tipo de estudo, o que já foi mencionado por Dessen (1995) quando afirmou que a concordância entre observadores de um mesmo fenômeno psicológico auxilia na medição da fidedignidade sobre essa observação. Contudo, deve-se atentar para que ambos os pesquisadores tenham conhecimento sobre 0 fenômeno estudado e os critérios estipulados no objetivo deste estudo.

O segundo ponto relevante a se destacar é a autoria coletiva com vinculação de pesquisadores de diferentes instituições, que amplia o caráter avaliativo dos resultados e aumenta as possibilidades de desdobramentos advindos de diferentes perspectivas teóricas. Para Lopes e Costa (2012) um dos pontos positivos sobre a coautoria em trabalhos científicos se deve à menor possibilidade de erros teóricos e metodológicos, uma vez que o estudo conta com a avaliação de terceiros. Ainda para esses autores, trabalhos em colaboração científica quando realizados de forma rigorosa, atentos às diretrizes pedagógicas da produção coautoral e que tenham o objetivo de realizar intercâmbios cientíicos, de caráter teórico e metodológico, são formas válidas de gerar conhecimentos.

\section{Objetivos dos estudos}

Os estudos localizados em periódicos internacionais tinham os seguintes objetivos: examinar as respostas vocais e gestuais contingentes dos bebês à linguagem e gestos das mães (Kuchirko, Tafuro, \& Lemonda, 2017); verificar as mudanças durante o primeiro ano em contingências sociais entre o olhar infantil, a exploração de objetos e a fala das mães (Chang, Barbaro, \& Deak, 2016); investigar as habilidades dos bebês para solicitar e informar com o olhar e o gesto de apontar (Bourdauis, Danis, Bacle, Santolini, \& Tijus, 2013); analisar a relação entre o mimetismo e o comportamento social dos bebês (Fawcett \& Liszkowski, 2012); e investigar a relação entre interação social entre bebê e adulto e o mimetismo em situação de brincadeira (Hall, Holler, \& Kidd, 2013).

As produções localizadas em periódicos nacionais tinham os seguintes objetivos: analisar e comparar a interação dirigida por pais aos bebês (Fuertes et al., 2017), identificar as habilidades sociocomunicativas de bebês, dirigidas às mães (Aquino \& Salomão, 2011a), analisar as manifestações de intencionalidade comunicativa em episódios interativos mãe-bebê, com o foco na interação das díades (Aquino \& Salomão, 2011b; Camargo, Salomão, Aquino, \& Nunes, 2015; Fuertes, Faria, Soares, \& Oliveira-Costa, 2010) e investigar o papel e as caracteristicas do desenvolvimento gestual no primeiro ano de vida (Nogueira, 2009).

É possivel perceber que os estudos sobre essa temática têm como objetivo, de maneira geral, buscar compreender a partir de que momento da vida o bebê começa a se expressar socialmente com base na cultura em que está inserido. Faz-se necessário salientar que nos estudos analisados, embora a brincadeira tenha comparecido em todos os cenários de pesquisa, sendo apontada como atividade favorecedora para a interação mãe-bebê, ela foi objeto de estudo apenas no artigo de Hall, Holler e Kidd (2013). Segundo esses autores, a brincadeira, o gesto e a linguagem demostram a natureza simbólica e social de nossa espécie. Especificamente, nesse estudo, postulou-se que as brincadeiras e os gestos permitem que o bebê externalize representações mentais compartilhadas entre criança e adulto em atividades conjuntas, o que ajuda a desenvolver a compreensão simbólica, que é um dos requisitos fundamentais para uma aquisição da linguagem.

\section{Delineamento das pesquisas, amostras e instrumentos}

Os estudos localizados em periódicos de âmbito nacional $(n=6)$ apresentaram estudos de caráter observacional, com amostras compostas por diades mãe-bebê (Aquino \& Salomão, 2011a; Aquino \& Salomão, 2011b; Camargo, Salomão, Aquino, \& Nunes, 2015; Fuertes, Faria, Soares, \& Oliveira-Costa, 2010) ou díades pai-bebê e mãe-bebê (Fuertes et al., 2017) observadas e videogravadas durante situações de brincadeira. Desses estudos, quatro eram de delineamento longitudinal (Aquino \& Salomão, 2011a; Aquino \& Salomão, 2011b; Camargo, Salomão, Aquino, 
\& Nunes, 2015; Nogueira, 2009) e dois de delineamento transversal (Fuertes et al., 2017; Fuertes, Faria, Soares, \& Oliveira-Costa, 2010). A maioria das videogravações $(n=4)$ ocorreu na residência dos pais dos bebês (Aquino \& Salomão, 2011a; Aquino \& Salomão, 2011b; Camargo, Salomão, Aquino, \& Nunes, 2015; Nogueira, 2009) e as demais ocorreram em uma instituição pública de saúde (Fuertes, Faria, Soares, \& Oliveira-Costa, 2010) ou pela escolha dos pais por suas residências ou um gabinete clínico (Fuertes et al., 2017).

No que se refere à quantidade da amostra, o estudo de Fuertes (2017) contou com 80 diades, sendo 40 pai-bebê e 40 mãe-bebê e os bebês tinham 15 meses de idade. O estudo de Fuertes, Faria, Soares e Oliveira-Costa (2010) analisou 99 díades mãe-bebê, de bebês de três a seis meses de idade. Já os estudos de Aquino e Salomão, (2011a), Aquino \& Salomão, (2011b) e Camargo, Salomão, Aquino e Nunes, (2015) contaram com seis diades mãe-bebê e os bebês foram observados longitudinalmente nas idades de seis, nove e 12 meses. Por fim, o estudo de Nogueira (2009) observou uma diade mãe-bebê, do nascimento até os 12 meses de idade do bebê. Além das videogravações, esses estudos utilizaram cronômetro, lápis e papel como instrumentos de pesquisa.

Os estudos localizados em periódicos de âmbito internacional $(n=5)$ apresentaram pesquisas experimentais, com amostras compostas por diades mãe-bebê (Bourdauis, Danis, Bacle, Santolini, \& Tijus, 2013; Chang, Barbaro, \& Deak, 2016; Kuchirko, Tafuro, \& Lemonda, 2017) ou bebêpesquisadoras(es), embora os pais estivessem no cenário (Fawcett \& Liszkowski, 2012; Hall, Rumney, Holler, \& Kid, 2013), videogravadas em situação de brincadeira. Desses estudos, dois eram de delineamento longitudinal (Chang, Barbaro, \& Deak, 2016; Kuchirko, Tafuro, \& Lemonda, 2017) e três eram de delineamento transversal (Bourdauis, Danis, Bacle, Santolini, \& Tijus, 2013; Fawcett \& Liszkowski, 2012; Hall, Rumney, Holler, \& Kidd, 2013). Na maioria dos estudos as videogravações ocorreram na residência dos pais dos bebês (Bourdauis, Danis, Bacle, Santolini, \& Tijus, 2013;
Chang, Barbaro \& Deak, 2016; Hall, Rumney, Holler, \& Kidd, 2013; Kuchirko, Tafuro, \& Lemonda, 2017) e um dos estudos não especificou o local onde a videogravação ocorreu (Fawcett \& Liszkowski, 2012).

No que se refere à quantidade da amostra, o estudo de Kuchirko, Tafuro e Lemonda (2017) contou com 190 diades mãe-bebê, que foram videogravadas quando os bebês tinham 14 e 24 meses de idade. O estudo de Bourdauis, Danis, Bacle, Santolini e Tijus, (2013) contou com uma amostra de 20 diades mãe-bebê, cujos bebês foram divididos em dois grupos de idades: 10 bebês com idade de 10 meses e 15 dias de vida e 10 bebês com idade de 13 meses e 15 dias de vida. O estudo de Chang. Barbaro e Deak, (2016) contou com uma amostra de 42 diades mãe-bebê, que foram videogravadas quando os bebês tinham as idades de quatro, seis e nove meses de vida. O estudo de Fawcett e Liszkowski (2012) teve uma amostra de 64 bebês de 18-19 meses de idade, divididos igualmente em dois experimentos ( 32 bebês no primeiro e 32 bebês no segundo). Por fim, o estudo de Hall, Rumney, Holler e Kidd (2013) apresentou uma amostra de 50 bebês de 18 a 31 meses de idade. Todos os estudos tiveram como instrumentos lápis, papel, cronômetro, brinquedos e câmera de vídeo. Além de vídeogravações, o estudo de Hall, Rumney, Holler e Kidd (2013) utilizou inventários para averiguar vocabulário e gestos dos bebês.

Para Dessen (1995, p. 223) "os estudos recentes sobre interações e relações sociais que empregam a metodologia observacional têm-se caracterizado, cada vez mais frequentemente, pela utilização de vídeo para a coleta de dados". A utilização de vídeos em pesquisas observacionais é importante, uma vez que permite ao pesquisador, no momento de transcrição e posterior análise da gravação, uma reflexão e reconstrução ativa do conteúdo gravado, que extrapola o seu campo de alcance cognitivo pela confrontação entre o registrado e o observado in loco.

Percebe-se que os estudos selecionados apresentam uma variabilidade de delineamentos de pesquisa para investigar um mesmo fenômeno, o que possibilita ampliar o entendimento sobre 
o papel da brincadeira como favorecedora da habilidade de comunicação intencional. Vale salientar, ainda, que a maioria dos estudos teve como foco a observação da relação diade mãebebê, em suas próprias residências. Para Aquino e Salomão (2011a) nas primeiras interações mãe-bebê surgem significados compartilhados que são demonstrados a partir de gestos e vocalizações dos bebês dirigidos às mães, "que os recebe e, simultaneamente, dirige ao bebê sons e gestos (p. 336)". Neste sentido, justificase a importância de estudar essa díade como forma de compreender o desenvolvimento de habilidades sociocomunicativas iniciais infantis.

\section{Risco de viés dos estudos selecionados}

Segundo Almeida e Goulart (2017), revisões sistemáticas apresentam uma grande vantagem no que diz respeito ao conhecimento sobre como determinado tema vem sendo estudado no campo científico. No entanto, alguns tipos de pesquisa, a exemplo de estudos observacionais, apresentam uma maior vulnerabilidade no que diz respeito ao risco de viés. O viés inclui todas as distorções que podem ser identificadas em qualquer tipo de delineamento de pesquisa. Nesse sentido, foi elaborado um quadro de risco de viés por meio do instrumento Risk Of Bias In Non-randomized Studies - of Interventions (ROBINS-I). Essa é uma ferramenta Cochrane para avaliação da qualidade metodológica dos estudos observacionais de forma a analisar possiveis erros aleatórios no desenho dos estudos localizados. O risco de viés dos estudos selecionados foi avaliado a partir dos seguintes domínios: confundimento, se refere à existência de potenciais confundidores (variáveis associadas ao fator em estudo e ao desfecho) e análise adequada; seleção dos participantes, aponta se a amostragem dos participantes apresenta viés de seleção; aferição da intervenção (exposição), mensuração da ocorrência da (exposição) e confiança nessa informação; não recebimento da intervenção atribuida, viés de performance e contaminação entre os grupos. Avalia se os individuos de um determinado grupo efetivamente receberam as intervenções para as quais foram analisadas, bem como potenciais cointervenções; perdas, indicação do percentual de perdas e características de indivíduos com dados faltantes em comparação aos com seguimento completo; aferição nos desfechos, forma de mensuração da ocorrência dos desfechos e a confiança nessa informação; e relato seletivo dos desfechos, que indica a maior probabilidade de relato dos desfechos com resultados significativos.

O Quadro 1 apresenta o risco de viés dos estudos analisados considerando os sete domínios indicados. 
Quadro 1- Análise do risco de viés dos estudos selecionados

\begin{tabular}{|c|c|c|c|c|c|c|c|}
\hline $\begin{array}{l}\text { Autores e ano dos estudos } \\
\text { selecionados }\end{array}$ & 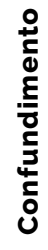 & 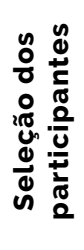 & 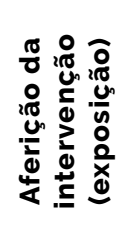 & 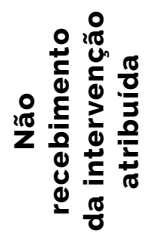 & 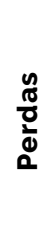 & 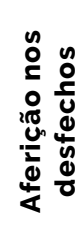 & 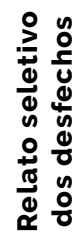 \\
\hline \multicolumn{8}{|l|}{ Nogueira (2009) } \\
\hline \multicolumn{8}{|l|}{$\begin{array}{l}\text { Fuertes; Faria; Soares \& Oliveira- } \\
\text {-Costa (2010) }\end{array}$} \\
\hline \multicolumn{8}{|l|}{ Aquino \& Salomão (2011a) } \\
\hline \multicolumn{8}{|l|}{ Aquino \& Salomão (2011b) } \\
\hline \multicolumn{8}{|l|}{ Fawcett \& Liszkowski (2012) } \\
\hline \multicolumn{7}{|l|}{ Hall; Rumney; Holler \& Kidd (2013) } & \\
\hline \multicolumn{8}{|l|}{$\begin{array}{l}\text { Bourdais; Danis; Bacle; Santolini \& } \\
\text { Tijus (2013) }\end{array}$} \\
\hline \multicolumn{8}{|l|}{$\begin{array}{l}\text { Camargo; Salomão; Aquino \& Nunes } \\
\text { (2015) }\end{array}$} \\
\hline \multicolumn{8}{|l|}{ Chang; Barbaro \& Deák (2016) } \\
\hline Fuertes et al. (2017) & & & & & & & \\
\hline Kuchirko; Tafuro \& LeMonda. (2017) & & & & & & & \\
\hline
\end{tabular}

Legenda:

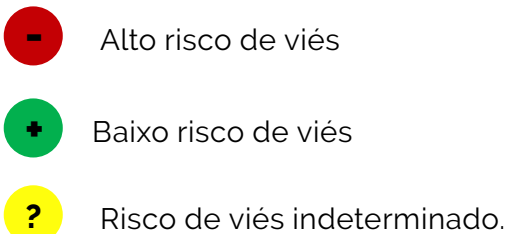

Como é possivel observar no Quadro 1, os estudos apresentam baixo risco de viés na maioria dos domínios avaliados. O item seleção dos participantes foi o que apresentou maior risco de viés nos estudos em função de selecionar seus participantes por conveniência.
Principais resultados dos estudos selecionados

Os estudos revelaram em seus resultados que a partir da observação de situações de brincadeira livre entre mãe e bebê é possivel identificar marcos na trajetória evolutiva e diferentes configurações da habilidade de comunicação intencional dos bebês. 
Nogueira (2009) ao investigar a linguagem gestual no primeiro ano de vida em uma diade mãe-bebê, afirmou que, com o desenvolvimento do bebê, há um aumento no tempo de engajamento conjunto com a mãe e com objetos de forma coordenada. A respeito da capacidade de responder reciprocamente às mães em situação de brincadeira, Fuertes et al. (2010) observaram nos bebês respostas faciais, vocais e afetivas e ressaltaram que os bebês apresentaram receptividade às solicitações maternas, indicando uma forte associação entre a sensibilidade materna e a cooperação infantil.

A partir da análise das habilidades sociocomunicativas evidenciadas por bebês em três momentos do primeiro ano de vida e suas diferentes configurações em cada idade, Aquino e Salomão (2011a) evidenciaram que é possivel identificar, principalmente aos 12 meses, a existência de habilidades comunicativas intencionais. As mesmas autoras ao observarem episódios de interação mãe-bebê em situação de brincadeira livre, verificaram que as aquisições de comunicação intencional redirecionam o curso das interações, em cada idade (Aquino \& Salomão, 2011b).

Também em situação de brincadeira livre entre mães e bebês com 18 meses de vida. Fawcett e Liszkowski (2012) apontaram que os bebês realizam durante a brincadeira o mimetismo como uma ferramenta para estabelecer conexões sociais com os outros e que o mimetismo tem influências especificas sobre os comportamentos sociais relacionados ao início das interações conjuntas subsequentes.

Já no tocante às inter-relações de desenvolvimento entre o brincar, uso de gestos e desenvolvimento da linguagem falada, Hall, Rumney, Holler e Kidd (2013), ao observarem diades mãe-bebê com 18 a 31 meses afirmaram que o jogo simbólico é associado positivamente com o uso de gestos que, por sua vez, está positivamente associado ao conhecimento da linguagem falada. Também Bourdais, Danis, Bacle, Santolini e Tijus (2013) investigaram as habilidades de bebês de 10 e 13 meses para solicitar e informar olhando e apontando para as mães por meio de um estudo experimental utilizando situações de brincadeira estruturada. Os autores indicaram que durante a brincadeira os bebês aprendem o vinculo de ver-saber e não ver, não saber, o elo entre significante e significado, referentes, crenças e palavras. Afirma-se também que os bebês usam o olhar como um meio para indicar intencionalmente o que desejam.

Ainda sobre a comunicação mãe-bebê em situação de brincadeira, Camargo, Salomão, Aquino e Nunes (2015) investigaram a interação de díades na fase pré-verbal dos bebês e identificaram que especialmente aos 9 meses ocorrem mudanças na comunicação pré-verbal, de modo que o gesto dêitico de alcançar deixa de ser única categoria utilizada pela criança e passa a ser substituído pelo gesto de apontar, ou seja, o repertório comunicativo intencional da criança vai se ampliando. As autoras constataram, ainda, que os gestos dêiticos mais utilizados no estabelecimento e manutenção de episódios interativos foram o de alcançar, por parte dos bebês, e o de mostrar, por parte das mães. Observou-se que mudanças nos gestos das crianças ao longo do seu desenvolvimento foram acompanhadas por mudanças nos gestos maternos.

Acerca das mudanças durante o primeiro ano de vida em contingências sociais entre o olhar infantil, a exploração de objetos e a fala das mães, Chang, Barbaro e Deak (2016) investigaram as ações do olhar e da mão dos bebês e das mães e a nomeação de objetos e enunciados gerais das mães. Durante a situação de brincadeira livre observou-se que as mães chamavam mais os objetos quando as crianças vocalizavam, olhavam para objetos ou para o rosto da mãe ou lidavam com vários objetos. À medida que os bebês se desenvolviam, sua crescente exploração de objetos criava oportunidades para as contingências da mãe e as modificava, bem como o olhar e as mãos passavam a acompanhar a nomeação de objetos ao longo do tempo.

Em um estudo mais recente, Kuchirko, Tafuro \& Lemonda (2017) examinaram respostas vocais e gestuais de bebês à linguagem e gestos das mães durante situação de brincadeira e indicaram que os bebês vocalizaram e gesticularam seguindo 
a linguagem referencial das mães e os gestos. No citado estudo participaram 190 diades mãebebê méxico-americanas, dominicanas e afroamericanas quando os bebês tinham 14 e 24 meses. Os autores afirmaram que no nivel individual, os bebês responsivos tinham mães responsivas.

Conforme foi possivel verificar nos estudos acima citados, a brincadeira corresponde a um elemento favorecedor da habilidade de comunicação intencional infantil, uma vez que fornece meios para o desenvolvimento comunicativo, a exemplo do envolvimento da criança em contextos compartilhados intencionalmente. Os estudos apontaram a ocorrência de diferentes configurações de comunicação intencional infantil que emergem durante episódios de brincadeira, tais como o desenvolvimento dos gestos, do mimetismo, da cooperação infantil, do olhar coordenado do bebê e da percepção do elo entre significante e significado.

Salienta-se que todos os estudos que compuseram a presente revisão englobam a brincadeira enquanto cenário para o desenvolvimento de habilidades comunicativas intencionais infantis. No entanto, no estudo de Hall et al. (2013), a brincadeira é estudada enquanto objeto de pesquisa, sendo aferida e correlacionada positivamente com o uso de gestos e com a linguagem falada. Com base nos resultados do referido estudo, os pesquisadores sugeriram outros caminhos para pesquisas futuras com o tema, a exemplo de estudos longitudinais para fortalecer as associações encontradas na amostra, como também a ampliação da amostra para outras culturas, uma vez que as atitudes para com a brincadeira e os estilos interacionais diferem entre as culturas.

\section{Considerações finais}

Esta revisão sistemática teve como objetivo identificar estudos que abordassem a brincadeira como promotora da habilidade de comunicação intencional infantil. Observou-se nos estudos analisados que a brincadeira é um importante cenário no qual se pode identificar comportamentos comunicativos intencionais e contribuir para o desenvolvimento da intencionalidade compartilhada. Ressalta-se também que a brincadeira pode ser fundamental para impulsionar o desenvolvimento de habilidades tipicamente humanas que precedem e acompanham a linguagem oral, tal como a habilidade sociocomunicativa intencional.

Embora a brincadeira tenha sido abordada nos estudos selecionados como cenário propício para o desenvolvimento de habilidades sociocomunicativas, nota-se a ausência de pesquisas que informem relatos sobre os tipos de brincadeira que podem ser realizadas ou propostas intencionalmente com o objetivo de favorecer o desenvolvimento humano inicial, tanto em espaços de interação informais quanto em ambientes formais de educação infantil, tais como berçários e creches.

A análise das pesquisas que abordaram a relação imbricada entre intencionalidade comunicativa e brincadeira nos permite sugerir a realização de estudos longitudinais com crianças nos três primeiros anos de vida, período no qual as crianças demonstram formas de brincar simbólica ou representativa. Estudos microgenéticos e longitudinais que acompanhem o transcurso da habilidade de comunicação intencional e atenção compartilhada durante situações de brincadeira são importantes ainda por disponibilizarem dados empíricos que possam auxiliar profissionais como psicólogos e educadores a elaborar programas de intervenção precoces direcionados a crianças com prejuizos na comunicação, na capacidade de simbolizar e de estabelecer trocas intersubjetivas. A relevância dessas habilidades para 0 desenvolvimento global infantil e da linguagem acentua a necessidade de realização de pesquisas do campo da Psicologia do Desenvolvimento em espaços formais de aprendizado, tais como as instituições de educação infantil que atendem crianças desde o primeiro ano de vida.

\section{Referências}

Almeida, C. P. B., \& Goulart, B. N. G. (2017). Como minimizar vieses em revisões sistemáticas de estudos observacionais. Revista CEFAC, 19(4), 551-555. https:// dx.doi.org/10.1590/1982-021620171941117. 
Aquino, F. S. B., \& Salomão, N. M. R. (2011a). Habilidades sociocomunicativas de bebês no primeiro ano de vida: um estudo longitudinal. Paidéia, 21(50), 335-344. (DOI INEXISTENTE).

Aquino, F. S. B., \& Salomão, N. M. R. (2011b). Intencionalidade comunicativa e atenção conjunta: uma análise em contextos interativos mãe-bebê. Psicologia: Reflexão e Critica, 24(1), 107-115. http://dx.doi.org/10.1590/ S0102-79722011000100013.

Bourdais, C., Danis, A., Bacle, C.,_Santolini, A., \& Tijous, C. (2013). Do 10- and 13-month-old infants provide informative gestures for their mothers in a hiding game? Infant Behavior \& Development, 36, 94-101. http://dx.doi. org/10.1016/j.infbeh.2012.11.006.

Camargo, J. F., Salomão, N. M. R., Aquino, F. S., \& Nunes, L. L. (2015). Os gestos na comunicação mãe-bebê: um estudo longitudinal. Estudos e pesquisas em psicologia, 15 (2), 652-670. (DOI INEXISTENTE).

Chang, L., de Barbaro, K., \& Deák, G. O. (2016). How do infants' actions affect when parents name objects? Developmental Neuropsychology, 41, 342-361. https:// doi.org / $/ 10.1080 / 87565641.2016 .1274313$

Cypel, S. (org) (2011) Fundamentos do desenvolvimento infantil: da gestação aos 3 anos. São Paulo: Fundação Maria Cecilia Souto.

Dessen, M.A. (1995). Tecnologia de vídeo: registro de interações sociais e cálculos de fidedignidade em estudos observacionais. Psicologia: Teoria e Pesquisa, (11) 3, 223-227. (DOI INEXISTENTE).

Fawcett C., Liszkowski U. (2012). Observation and initiation of joint action in infants. Child Dev. 83 (1), 434-441. 10.1111/j.1467-8624.2011.01717.x

Fuertes, M., Castro, S., Alves, M. J., Faria, A., Osório, B., \& Sousa, O. (2017). Interação e linguagem dirigida a crianças de quinze meses. Psicologia USP, 28(3), 346357. http://dx.doi.org/10.1590/0103-656420160154.

Fuertes, M., Faria, A., Soares, H., \& Oliveira-Costa, A. (2010). Momentos de interação em que as emoções se apre(e)ndem: estudo exploratório sobre a prestação materna e infantil em jogo livre. Psicologia USP, 21(4), 833-857. (DOI INEXISTENTE).

Hall, S., Rumney, L., Holler, J., Kidd, E. (2013). Associations among play, gesture and early spoken language acquisition. First Language, 33(3) 294-312. https://doi. org/10.1177/0142723713487618.

Hamann K, Warneken F, Tomasello M. (2012). Children's developing commitments to joint goals. Rev. Child Dev. 83(1). 137-45. 10.1111/j.1467-8624.2011.01695.x

Kuchirko, Y., Tafuro, L. \& LeMonda, C. S. T. (2017). Becoming a Communicative Partner: Infant Contingent Responsiveness to Maternal Language and Gestures. Infancy, 1-19. 10.1111/infa.12222.

Lopes. A. C. \& Costa, H. H. C. (2012). A produção bibliográfica em coautoria na área de educação. Revista Brasileira de Educação, 17 (51). 717-152. http://dx.doi. org /10.1590/S1413-24782012000300013.
Mendes, D. M.L.F. \& Seidl-de-Moura, M. L. (2004). Desenvolvimento da brincadeira e linguagem em bebês de 20 meses. Rev. Psicologia Teoria e Pesquisa. Set-Dez, 20(3), pp. 215-222. http://dx.doi.org/10.1590/S010237722004000300002 .

Moher, D., Liberati, A., Tetzlaff, J., Altman, D.G. \& The Prisma Group (2015). Preferred reporting items for systematic reviews and meta-analyses: the PRISMA statement. Epidemiol. Serv. Saúde, 24(2), 335-342 DOI: 10.5123/S1679-49742015000200017

Nogueira, S. E. (2009). A gênese da comunicação gestual e o desenvolvimento cognitivo: um estudo longitudinal. Tese de Doutorado. Programa de Pós-graduação em Psicologia Social. Universidade do Estado do Rio de Janeiro, Rio de Janeiro, RJ.

Papaeliou, C. F. \& Trevarthen, C. (2006). Prelinguistic pitch patterns expressing 'communication' and 'apprehension'. Journal of Child Language, 33, 163-178. DOI: 10.1017/S0305000905007300

Queiroz, N. L. N., Maciel, D. A. \& Branco, A. U. (2006) Brincadeira e desenvolvimento infantil: um olhar sociocultural construtivista. Rev. Paidéia 16(34), 169-179. http://dx.doi.org/10.1590/S0103-863X2006000200005.

Rivero, M. (2003). Los inícios de la comunicación: la intencionalidad comunicativa y el significado como procesos graduales. Anuário de Psicologia, 34(3), 337356. (DOI INEXISTENTE).

Rochat, P. (2007). Intentional action arises from early reciprocal exchanges. Acta Psychologica, 124, 8-25. http://dx.doi.org/10.1016/j.actpsy.2006.09.004

Santos, C. M. C., Pimenta, C. A. M., \& Nobre, M. R. C. (2007). A estratégia PICO para a construção da pergunta de pesquisa e busca de evidências. Revista Latino- $A$ mericana de Enfermagem, 15(3), 508-511. https://dx.doi. org/10.1590/S0104-11692007000300023.

Sarriá, E. (1991). Observacion de la comunicacion intencional preverbal: um sistema de codificacion basado em el concepto de categoria natural. Psicotema, 3(2), 359-380. (DOI INEXISTENTE).

Smirnova, E. O. \& Riabkova, I. A. (2016). Psychological Features of the Narrative-Based Play of Preschoolers Today. Journal of Russian \& East European Psychology, 53(2), pp. 40-55. https://doi.org/10.1080/10610405.2016.1230993

Striano, T. \& Vaish, A. (2006). Seven-to-9-month-old infants use facial expression to interpret others' actions. British Journal of Developmental Psychology, 24, 753-760. DOI: 10.1348/026151005X70319

Teodoro, W. L. G. (2013) O desenvolvimento infantil de o a 6 a vida pré-escolar. Uberlândia, 114p

Tomasello, M. (2003). Origens culturais da aquisição do conhecimento humano. (C. Berliner, Tad.). São Paulo: Martins Fontes. (Coleção Tópicos)

Tomasello, M. \& Carpenter, M. (2007). Shared intentionality. Development Science, 10(1), 121-125. DOI: 10.1111/j. 1467-7687.2007.00573.x

Vigotsky, L. S. (2004). Teoria e método em Psicologia. São Paulo-SP: Ed. Martins Fontes, $3^{\circ}$ ed. 
Villachan-Lyra, P., Queiroz, E, F. F. Moura, R. B. \& Gil, M. O. G. (2018). Entendendo o desenvolvimento infantil: contribuições das neurociências e o papel das relações afetivas para pais e educadores. Curitiba: Appris. 59 p.

\section{Agradecimento}

Pesquisa financiada pela Coordenação de Aperfeiçoamento de Pessoal de Nivel SuperiorCAPES

\section{Ana Cristina Ramos Costa}

Mestra em Psicologia Social pelo Programa de Pós-graduação em Psicologia Social da Universidade Federal da Paraiba (UFPB, João Pessoa, PB, Brasil).

\section{Vanessa Porto Alexandrino}

Doutoranda no Programa de Pós-graduação em Psicologia Social da Universidade Federal da Paraiba (UFPB, João Pessoa, PB, Brasil).

\section{Jéssica Andrade de Albuquerque}

Doutoranda no Programa de Pós-graduação em Psicologia Social da Universidade Federal da Paraiba (UFPB, João Pessoa, PB, Brasil)

\section{Fabíola de Sousa Braz Aquino}

Doutora em Psicologia Social pelo Programa de Pós- graduação em Psicologia Social da Universidade Federal da Paraiba (UFPB, João Pessoa, PB, Brasil), docente do Departamento de Psicologia da Universidade Federal da Paraíba (UFPB) e do Programa de Pós-graduação em Psicologia Social (UFPB) em João Pessoa, PB, Brasil.

\section{Endereço para correspondência}

Fabiola de Sousa Braz Aquino

Universidade Federal da Paraiba

Cidade Universitária, Centro de Ciências Humanas, ambiente 26

58051-900

João Pessoa, PB, Brasil 